\title{
The Friction on Cartilage Surfaces under Variable Wettability
}

\author{
Aleksandra Mrela ${ }^{1}$ and Zenon Pawlak ${ }^{2 *}$ \\ ${ }^{1}$ Kujawy and Pomorze University in Bydgoszcz, Faculty of Technology, USA \\ ${ }^{2}$ Tribochemistry Consulting, Salt Lake City, UT 84117, USA, University of Economy, Biotribology Laboratory, USA
}

Received: 㭗 June 25, 2018; Published: 非July 13, 2018

*Corresponding author: Zenon Pawlak, Tribochemistry Consulting, Salt Lake City, UT 84117, USA

\section{Abstract}

The wettability of the articular surface of cartilage depends on the condition of its surface-active phospholipid overlay, which is structured as a multi-bilayer. We examined the characteristics of this membrane surface entity in both its normal and degenerated conditions using a combination of atomic force microscopy, contact angle measurement, and friction test. The observations have led to the conclusions that the friction coefficient is significantly dependent on the hydrophobicity (wettability) of the surface of the tissue, thereby confirming the hypothesis tested in this paper.

Keywords: Articular Cartilage; Phospholipid Bilayer; Wettability; Atomic Force Microscopy; Hydrophilic; Hydrophobic

\section{Introduction}

Phospholipids are molecules present in various tissues and body fluids which are also named surfactants, substances which lower surface energy [1-3].The main phospholipid classes adsorbed onto the surface of cartilage surface-active phospholipids (SAL) were identified and quantified: phosphatidylcholine $(41 \%)$, phosphatidyl ethanol amine (27\%) and sphingomyelin (32\%) were identified as the major components of the lipid bilayer coating a natural intact cartilage surface [4,5]. The human body naturally produces phospholipids. Phospholipids support most functions of organs, such as cardiovascular health, nerve health, liver function, digestion and, most importantly, certain phospholipids might act as boundary lubricants [1].
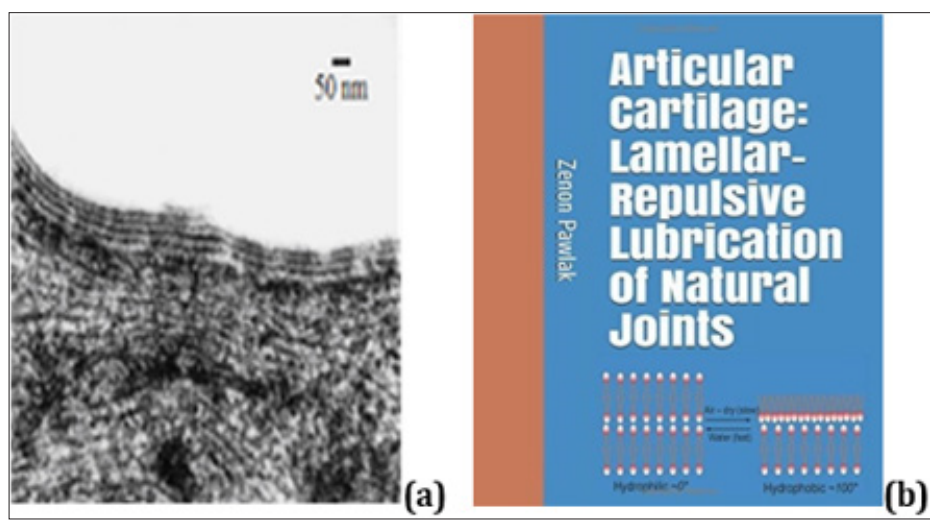

Figure 1: (a) An electron microscopy image of the articular cartilage surface of a human knee demonstrating the oligolamellar lining consisting of phospholipid bilayers [3]. The bar represents $50 \mathrm{~nm}$ and (b) Book cover "Articular cartilage: Lamellarrepulsive lubrication of natural joints" [1].

(Figure 1) (a) An electron microscopy image of the articular cartilage surface of a human knee demonstrating the oligo lamellar lining consisting of phospholipid bilayers [3]. The bar represents $50 \mathrm{~nm}$ and (b) Book cover "Articular cartilage: Lamellar-repulsive lubrication of natural joints" [1]. This smart surface characteristic creates a hydrophobic-hydrophilic balance resulting in a functional hydrophilic surface in the intact joint. One of the quantitative indicators of surface tribo chemical properties is wettability. This is measured as the contact angle between a drop of water and the reference surface, see (Figure 2a). 


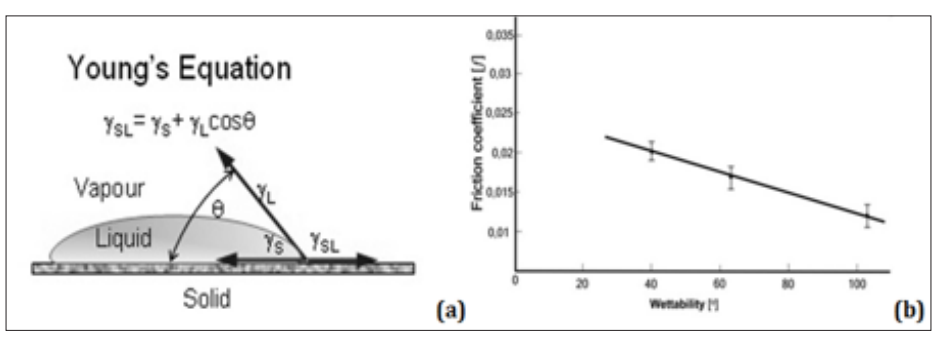

Figure 2: (a) Contact action for the wetting of a surface, where $\theta$ denote the contact angle, - the liquid free energy, - the solid free energy, - the solid/liquid interfacial free energy; (b) Coefficient of friction $(f)$ vs. wettability, $\sim 0 \bigotimes)$ of normal cartilage ( $\sim 103 \rrbracket)$ and gradually depleted $(\sim 65$ o and 39.1凶 and)cartilage surfaces measured in saline solution.

Wet ability characterizes the surface of various objects, which are generally defined as wettable (highly hydrophilic, $\sim 0 \bigotimes$ to $45 \rrbracket$ ) or non wettable (highly hydrophobic, $\sim 90 \rrbracket$ to $180 \bigotimes$ ) [2]. It is well known that polymers with hydrophobic entities (CH2- and CH3-) can be modified by oxygen-plasma-treatment to those carrying a hydrophilic group $(-\mathrm{OH})$. This modification makes the surface hydrophilic with a small contact angle, which is effective in full aqueous lubrication $[6,7]$.

(Figure 2a) Contact action for the wetting of a surface, where $\theta$ denote the contact angle, - the liquid free energy, - the solid free energy, - the solid/liquid interfacial free energy; (b) Coefficient of friction (f) vs. wettability, $\sim 0 \bigotimes)$ of normal cartilage $(\sim 103 \rrbracket)$ and gradually depleted ( $\sim 650$ and $39.1 \otimes$ and)cartilage surfaces measured in saline solution. In this paper, we examine using some methods, such as AFM, together with surface wettability measurements, a relationship between wettability and friction. The wettability of cartilage depends on the number of PLs bilayers acting as a solid lubricant; this hypothesis was tested with normal and depleted cartilage samples. AFM imaging was used to examine the multi bilayer cartilage surface.

\section{Materials and Methods}

The articular cartilage specimens were collected from bovine knees aged 15-20 months. Osteochondral plugs, of 5 and $10 \mathrm{~mm}$ in diameter, were harvested from lateral and medial femoral condyles using a circular stainless steel cutter. The cartilage discs were cut into $3 \mathrm{~mm}$ plugs with underlying bone. Two types of samples were tested: untreated bovine cartilage and bovine cartilage treated with a Folch reagent [8] (a 2:1 v/v mixture of chloroform and methanol), and a lipid-rinsing solution to remove the lipids from the surface of the cartilage. After preparation, the specimens were stored at 253 $\mathrm{K}$ in a $0.155 \mathrm{M} \mathrm{NaCl}(\mathrm{pH}=6.9)$ solution and fully defrosted prior to testing. The cartilage discs were then glued to the disc and pin stainless steel surfaces, and friction tests were conducted in the universal buffer solution.

Tensiometer: The contact angle between the liquid and the tested cartilage was measured using a KSV CAM100 tensiometer. The contact angle measured was that between a droplet of a $0.15 \mathrm{M}$ saline solution and a given air-dry cartilage surface. The contact angle test performed on the normal, partial and completely depleted cartilage samples. Five tests were performed on each specimen and each set-up.
Friction test: The measurements were performed using a sliding pin-on-disc tribotester T-11 manufactured by the NIST Research, Radom, Poland. The tests were conducted at room temperature, at a speed of $1 \mathrm{~mm} / \mathrm{s}$ during 5 minutes, and under a load of $15 \mathrm{~N}$ (1.2 MPa) which correspond to the physiological lubrication condition [9]. Prior to the friction tests, the lubricants were prepared using the Britton-Robinson buffer solution [10], and their $\mathrm{pH}$ values were measured. The friction coefficients measurements of cartilage/cartilage tribo pair were carried out over the $\mathrm{pH}$ range between 2.5 and 9.5.

\section{Results and discussion}

\section{The friction under variable wettability}

Wetting of different surfaces (S) with a drop of saline: (a) when placed on surfaces with contact angles ( ) greater than $150^{\circ}$, (b) when placed on normal (an intact, air-dried) bovine articular cartilage) $(, \sim 100 \rrbracket)$, and (c) when placed on an articular surface of a human hip diagnosed with osteoarthritis ( , 40囚). (Figure 3a) The friction coefficient of (AC)/(AC) vs. wettability of surface (AC): (1) bovine and human cartilage surface $103^{\circ}$ and $101^{\circ}$ (2) knee $79.7^{\circ}$ (3) unhealthy cartilage 65o, (4) knee $63^{\circ},(5)$ degenerated hip $56.3^{\circ}$, (6) bovine cartilage surface partially depleted $63^{\circ},(7)$ partially depleted $53^{\circ}$ and (8) completely depleted surface $35.1^{\circ}$ [4, 11]. The wettability of cartilage surface was measured the under an air-dry condition at room temperature. (Figure $3 b$ ) The variation of wettability of AC vs the number of phospholipid bilayers. Note the typical inter lamellar aqueous spacing of $4.5 \mathrm{~nm}$ between bilayers [1]. Figure 3.3.5 in [1] represents the coefficient of friction vs. time for the (cartilage-cartilage) pair for normal and dilapidated surfaces. These friction tests thus confirm our hypothesis on the relation between the number of phospholipids bilayers (or wettability) and friction [12-16].

For normal $(\mathrm{AC} / \mathrm{AC})$ pairs the range of $\mathrm{f}=0.004-0.012$, and in the ranges of $f=0.011-0.09$ unhealthy cartilage, and 0.015 0.024 for the depleted (AC-AC) pairs. This reveals the significantly higher friction coefficients between (AC-AC) pairs in the depleted conditions relative to those of the normal (AC-AC) pairs with intact contacting surfaces. The ratio of the coefficient of friction for the depleted (AC-AC) pair/normal (AC-AC) pair values after 5 min increased 2.3 and 3.0 times for the depleted (AC-AC) pairs. (Figure 2b) represents the plot of coefficient of friction ( $f$ ) vs hydrophobicity (wettability) for normal and dilapidated cartilage surface samples after $5 \mathrm{~min}$ of a test. The frictional characteristics 
of the treated cartilage were greatly increased (over 200\%) relative to that of the untreated cartilage surface. Furthermore, it has been shown that the phospholipid on the surface of AC as bilayers is a lubricant and supports lubrication mechanism by lowering the friction of bio surfaces [17].

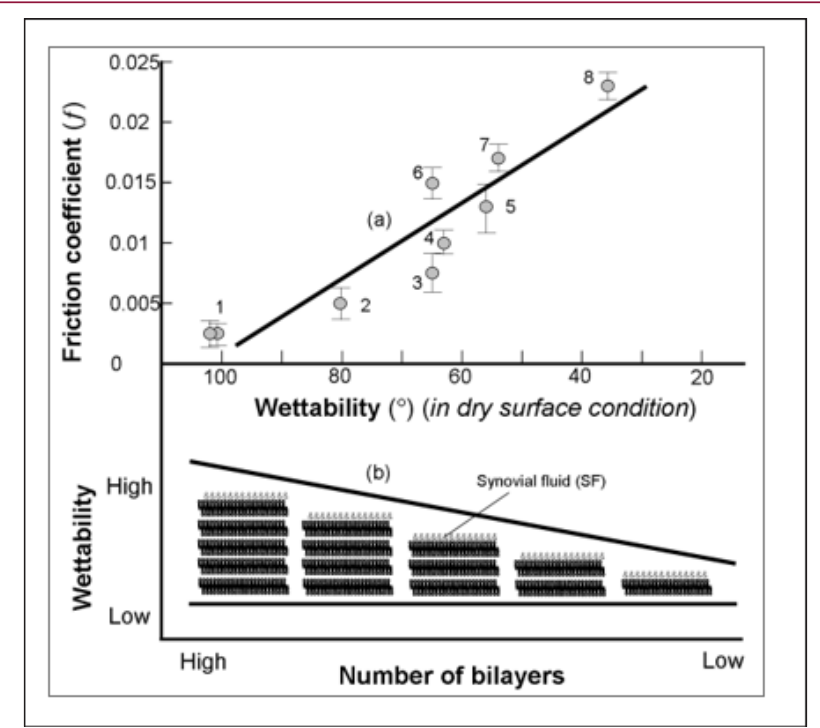

Figure 3: (a) The friction coefficient of (AC)/(AC) vs. wettability of surface (AC): (1) bovine and human cartilage surface $103^{\circ}$ and $101^{\circ}(2)$ knee $79.7^{\circ}$ (3) unhealthy cartilage 65o, (4) knee $63^{\circ}$, (5) degenerated hip $56.3^{\circ}$, (6) bovine cartilage surface partially depleted $63^{\circ}$, (7) partially depleted $53^{\circ}$ and (8) completely depleted surface $35.1^{\circ}[4,11]$. The wettability of cartilage surface was measured the under an air-dry condition at room temperature. (b) The variation of wettability of AC vs. the number of phospholipid bilayers. Note the typical interlamellar aqueous spacing of $4.5 \mathrm{~nm}$ between bilayers [1].

In (Figure 3a) (cartilage/cartilage) pairs were used to study the friction on cartilage surfaces under variable wettability. With the lipid-depleted cartilage samples and untreated normal samples, we were able to support observations made by other authors $[4,16,9]$.Our results demonstrated that wettability is an important factor for characterizing biological frictional surfaces. The friction coefficient vs. changes of wettability for natural joints with healthy and naturally degenerated articular surfaces [4] and bovine samples for partial and completely depleted cartilage samples, (Figure 3a \& Figure $3 b$ ) shows changes in wettability of AC surface vs. numbers of bilayers. We interpret the increased friction coefficient values in terms of the number of bilayers available in the SAL $[1,4,9]$. Both friction and wettability show very similar behavior when the SAL thickness is varied $[2,16,18]$. The implication of this on cartilage has been observed during osteoarthritis where increased friction coefficient is measured due to gradual loss of the SAL $[12,19,20]$. Phospholipidic lamellar aggregates and bio macro molecules in SF may contribute to electrostatic hydration-repulsion during lubrication. The highly hydrated PL lamellar aggregates are expected to cover cartilage surfaces and support hydrophilic lamellarrepulsive hydration lubrication [20, 21].The results obtained from atomic force microscopy (AFM) imaging and characterization of the surface of normal intact and depleted articular cartilage specimens are presented in (Figure 4) Topographical (a, e) and deflection (b, f) 2-D images of articular cartilage surface (Frame size: 8 by $8 \mu \mathrm{m}$ ). Normal articular surface (a) and after a 21-min delipidization in chloroform/methanol (f). Wettability (a) 103o and (f) 39.10.

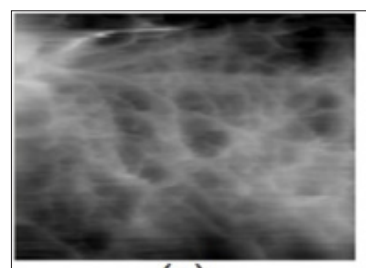

(a)

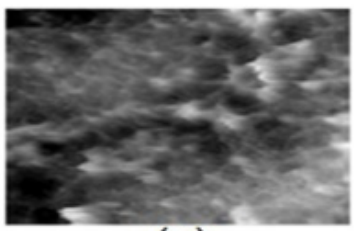

(e)

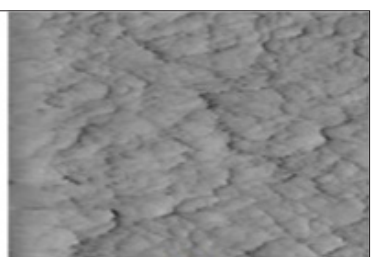

(b)

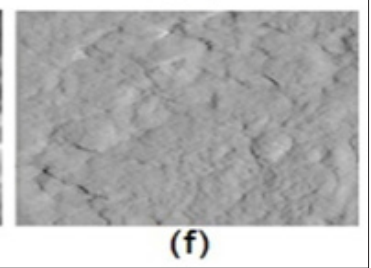

Figure 4: Topographical $(\mathrm{a}, \mathrm{e})$ and deflection $(\mathrm{b}, \mathrm{f})$ 2-D images of articular cartilage surface (Frame size: 8 by $8 \mu \mathrm{m})$. Normal articular surface (a) and after a 21-min delipidization in chloroform/methanol (f). Wettability (a) 103o and (f) 39.1o. 


\section{Conclusion}

We have examined natural articular cartilage surface and shown the relation between surface wettability and friction. Tests were performed to measure the wettability of normal and degraded bio surfaces, while also measuring the coefficients of friction of contacting cartilage surfaces. The number of PLs bilayers influences both wettability and surface friction, which are fundamentally important factors in joint lubrication. The key to understanding the mechanism of joint lubrication lies in obtaining insight into the relationships between the structure and function of surface bilayers.

\section{References}

1. Hills BA (1988) The Biology of Surfactant, Cambridge University Press, London.

2. Pawlak Z (2018) Articular Cartilage: Lamellar-Repulsive Lubrication of Natural Joints, Kindle Direct Publishing PP: 171.

3. Hills BA (2002) Surface-active phospholipid: a Pandora's box of clinical applications. Part II Barrier and lubricating properties. Int. Med. Journ 32(5-6): 242-251.

4. Hills BA, Monds MK (1998) Deficiency of lubricating surfactants lining the articular Surfaces of replaced hips and knees. British Journal of Rheumatology 37(2): 143-147.

5. Sarma AV, Powell GL and LaBerg M (2001) Phospholipid composition of articular cartilage boundary lubricant. Journal of Orthopedic Research 19(4): 671-676.

6. Bongaerts JHH, Fourtouni K and Stokes JR (2007) Soft-tribology: lubrication in a ompliant PDMS-PDMS contact. Tribology International 40(10-12): 1531-1542.

7. Lee S and Spencer ND (2005) Aqueous lubrication of polymers: Influence of surface modification. Tribology International 38(11-12): 922-930.

\section{ISSN: 2574-1241}

DOI: 10.26717/BJSTR.2018.06.001404

Zenon Pawlak. Biomed J Sci \& Tech Res

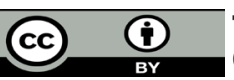

This work is licensed under Creative Commons Attribution 4.0 License

Submission Link: https://biomedres.us/submit-manuscript.php
8. Folch J, Lees M, and Sloane-Stanley GH (1957) A simple method for the isolation and purification of total lipids from animal tissues. Journal of Biological Chemistry 226(1): 497-509.

9. Pawlak Z, Urbaniak W, Oloyede A (2013) Natural articular joints: Model of a lamellar-roller bearings lubrication and the nature of the cartilage surface. Book: Biomaterials and Medical Tribology: Research and Development in Wood head Publishing Reviews: Mechanical Engineering Series.

10. Britton HTK, Robinson RA (1931) Universal buffer solutions and the dissociation constant of veronal. Journal of the Chemical Society pp. 1456-1462.

11. Pawlak Z, Yusuf KQ, Pai R, Urbaniak W (2017) Alamellar-repulsive mechanism of lubrication of natural joints. Archives Biochemistry and Biophysics 623-624: 42-48.

12. Higaki H, Murakami T, Nakanishi Y, Miura H, Mawatari T et al. (1998) The lubricating ability of biomembrane models with dipalmitoyl phosphati dylcholine and $\gamma$ globulin', Proceedings of the Institution of Mechanical Engineers. Part H: Journal of Engineering in Medicine 212(5): 337-346.

13. Pawlak Z, Urbaniak W, Gadomski A, Yusuf, KQ Afara IO et al. (2012) The role of lamellate phospholipid bilayers in lubrication of joints. Acta of Bioengineering and Biomechanics 14(4): 101-106.

14. Murakami T, Higaki H, Sawae Y, Ohtsuki N, Moriyama S et al. (1998) Adaptive multimode lubrication in natural synovial joints and artificial joints, Proceedings of the Institution of Mechanical Engineers. Part H: J. of Engineering in Medicine 212(1): 23-35.

15. Trunfio S farghiu AM, Berthier Y, Meurisse MH, Rieu JP (2008) Role of nano mechanical propertiesin the tribological performance of phospholipid biomimetic surfaces. Langmuir 24(16): 8765-8771.

16. Ozturek HE, Stoffel KK, Jones CF, Stachowiak GW (2004) The effect of surface-active phospholipids on the lubrication of osteoarthritic sheep knee joints: Friction. Tribology Letters 16: 283-289.

17. Greene GW, Banquy X, Woog LD, Lowrey DD, Yu J, et al. (2011) Adaptive mechanically controlled lubrication mechanism found in articular joints.

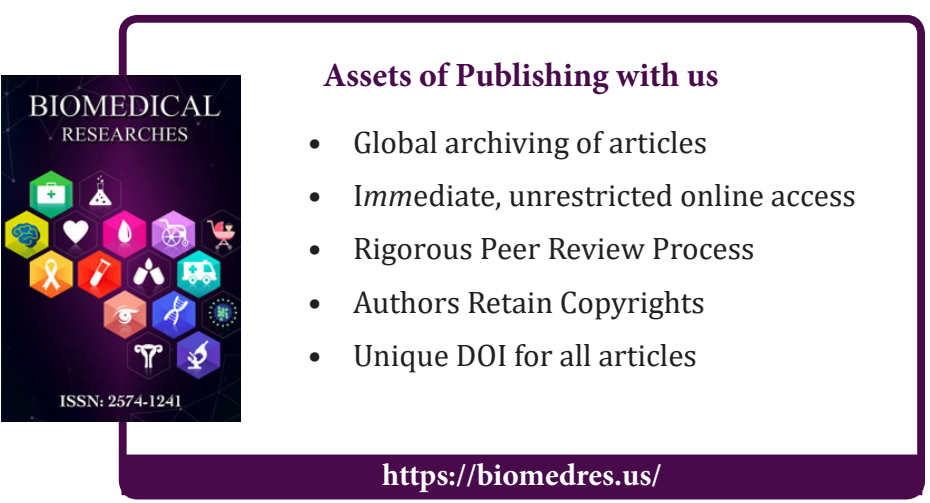

